OPEN ACCESS

Edited by:

Garret Suen,

University of Wisconsin-Madison, USA

Reviewed by:

Hilary G. Morrison,

Marine Biological Laboratory, USA

Georgios Oikonomou,

University of Liverpool, UK

Pamela L. Ruegg,

University of Wisconsin, Madison,

USA

*Correspondence: Sergine Even

sergine.even@rennes.inra.fr

${ }^{\dagger}$ Present Address:

Damien S. Bouchard,

ANSES-ANMV, Fougères, France

${ }^{*}$ These authors have contributed equally to this work.

Specialty section:

This article was submitted to

Systems Microbiology,

a section of the journal

Frontiers in Microbiology

Received: 24 November 2015

Accepted: 22 March 2016

Published: 08 April 2016

Citation:

Falentin $H$, Rault L, Nicolas A, Bouchard DS, Lassalas J, Lamberton P, Aubry J-M, Marnet P-G, Le Loir Y and Even S (2016) Bovine Teat Microbiome Analysis Revealed

Reduced Alpha Diversity and Significant Changes in Taxonomic Profiles in Quarters with a History of Mastitis. Front. Microbiol. 7:480. doi: 10.3389/fmicb.2016.00480

\section{Bovine Teat Microbiome Analysis Revealed Reduced Alpha Diversity and Significant Changes in Taxonomic Profiles in Quarters with a History of Mastitis}

\author{
Hélène Falentin ${ }^{1,2 \neq}$, Lucie Rault ${ }^{1,27}$, Aurélie Nicolas ${ }^{1,2}$, Damien S. Bouchard ${ }^{1,2 \dagger}$, \\ Jacques Lassalas ${ }^{3,4}$, Philippe Lamberton ${ }^{3,4}$, Jean-Marc Aubry ${ }^{3,4}$, Pierre-Guy Marnet ${ }^{3,4}$, \\ Yves Le Loir ${ }^{1,2}$ and Sergine Even ${ }^{1,2 *}$ \\ ${ }^{1}$ Institut National de la Recherche Agronomique, UMR 1253 STLO, Rennes, France, ${ }^{2}$ Agrocampus Ouest, UMR 1253 STLO, \\ Rennes, France, ${ }^{3}$ Institut National de la Recherche Agronomique, UMR 1348 PEGASE, Saint-Gilles, France, ${ }^{4}$ Agrocampus \\ Ouest, UMR 1348 PEGASE, Rennes, France
}

Mastitis is a mammary gland inflammatory disease often due to bacterial infections. Like many other infections, it used to be considered as a host-pathogen interaction driven by host and bacterial determinants. Until now, the involvement of the bovine mammary gland microbiota in the host-pathogen interaction has been poorly investigated, and mainly during the infectious episode. In this study, the bovine teat microbiome was investigated in 31 quarters corresponding to 27 animals, which were all free of inflammation at sampling time but which had different histories regarding mastitis: from no episode of mastitis on all the previous lactations (Healthy quarter, $\mathrm{Hq}$ ) to one or several clinical mastitis events (Mastitic quarter, Mq). Several quarters whose status was unclear (possible history of subclinical mastitis) were classified as NDq. Total bacterial DNA was extracted from foremilk samples and swab samples of the teat canal. Taxonomic profiles were determined by pyrosequencing on 16s amplicons of the V3-4 region. $\mathrm{Hq}$ quarters showed a higher diversity compared to Mq ones (Shannon index: 8 and 6 , respectively). Clustering of the quarters based on their bacterial composition made it possible to separate $\mathrm{Mq}$ and $\mathrm{Hq}$ quarters into two separate clusters ( $\mathrm{C} 1$ and C2, respectively). Discriminant analysis of taxonomic profiles between these clusters revealed several differences and allowed the identification of taxonomic markers in relation to mastitis history. C2 quarters were associated with a higher proportion of the Clostridia class (including genera such as Ruminococcus, Oscillospira, Roseburia, Dorea, etc.), the Bacteroidetes phylum (Prevotella, Bacteroides, Paludibacter, etc.), and the Bifidobacteriales order (Bifidobacterium), whereas C1 quarters showed a higher proportion of the Bacilli class (Staphylococcus) and Chlamydiia class. These results indicate that microbiota is altered in udders which have already developed mastitis, even far from the infectious episode. Microbiome alteration may have resulted from the infection itself and or the associated antibiotic treatment. Alternatively, differences in 
microbiome composition in udders with a history of mastitis may have occurred prior to the infection and even contributed to infection development. Further investigations on the dynamics of mammary gland microbiota will help to elucidate the contribution of this endogenous microbiota to the mammary gland health.

Keywords: bovine microbiota, bovine microbiome, dysbiosis, mammary gland, dairy ruminant, mastitis

\section{INTRODUCTION}

For decades now, the development of infections has been considered as the result of a bipartite interaction of a pathogen with a host. Development of high-throughput sequencing techniques has opened a new field of investigation that has made it possible to characterize microbiomes associated with hosts in greater depth and that has revealed a larger role than previously imagined for microbiota (Vayssier-Taussat et al., 2014). In animals, interest has increased in the exploration of microbiotas, notably for livestock species such as pigs, cattle and chickens, with regard to animal performance, genetics, diet and health (Sandri et al., 2014; Kim and Isaacson, 2015; Schokker et al., 2015; Weimer, 2015).

In cattle, major efforts have been devoted to the characterization of the rumen microbiome in the last few years in relation to diet, rumen development, fermentation efficiency including the production of greenhouse gases such as $\mathrm{CO} 2$ and methane, and animal performance (feed efficiency, milk composition; Jami et al., 2014, p. 2; McCann et al., 2014; Mohammed et al., 2014; Sandri et al., 2014; Henderson et al., 2015; Jewell et al., 2015; Kumar et al., 2015; Minuti et al., 2015; Myer et al., 2015; Veneman et al., 2015; Weimer, 2015). Two other microbiota have been explored, which are associated with the mammary gland and reproductive tract (uterus/vagina), in relation to the two main post-partum diseases in cattle, mastitis and metritis (Santos et al., 2011; Machado et al., 2012; Oikonomou et al., 2012, 2014; Santos and Bicalho, 2012; Knudsen et al., 2014; Rodrigues et al., 2015). Mastitis is an inflammation of the mammary gland that generally has an infectious origin. This inflammation is diagnosed through an increase of the somatic cell count (SCC) in milk, which mainly corresponds to neutrophil recruitment in the mammary gland. SCC increase can be associated with clinical signs including udder inflammation, abnormal milk or systemic signs such as fever (clinical mastitis) or the lack of clinical signs (subclinical mastitis). Mastitis is among the most common diseases in dairy cattle and affects animal welfare as well as productivity parameters (NaderMacías et al., 2008). It has a huge impact on the economy of the dairy production chain (Heikkilä et al., 2012). Prevention and curative strategies that mainly rely on antibiotherapy are not fully effective at this time, frequently resulting in chronic and recurrent infections. This prompts the need of a better understanding of host (mammary gland)/pathogen interactions as a prerequisite for the development of efficient diagnostic tools and therapeutic interventions. In line with the concept of pathobiome (Vayssier-Taussat et al., 2014), this better understanding of the host pathogen interaction includes a better characterization of the mammary gland microbiome and of its role in disease development.
So far, the analysis of mammary gland microbiota was mainly undertaken to provide new insights into the ecology of species related to inflammatory disorders, by comparing the microbiomes of healthy cows with the microbiomes of cows undergoing inflammation (Oikonomou et al., 2012, 2014; Kuehn et al., 2013). Using high-throughput automated DNA pyrosequencing, these studies have explored the bacterial profile of milk isolated from quarters undergoing clinical and subclinical mastitis and from healthy quarters, giving new insights into the bacterial profiles associated with mastitis, notably for culturenegative samples. It can be observed that the above-mentioned studies shed light on milk microbiota composition at the time of infection. Apart from the infectious episode, the composition of the microbiota of quarters that develop mastitis has never been explored.

In the present study, we used high-throughput automated DNA pyrosequencing to explore the link between bovine teat microbiome composition and history of quarter with regard to mastitis. Sampling was performed on quarters that were free of mastitis at the time of sampling (no signs of inflammation and low SCC), but that had different histories with regard to mastitis. We focused on the microbiota located inside the teat (contained in foremilk and attached to the teat internal epithelium) since this microbiota constitutes the first potential microbiological barrier against pathogen entry.

\section{MATERIALS AND METHODS Experimental Design}

Sampling was performed at the INRA UMR PEGASE experimental farm on a Prim'Holstein herd. The cows were kept indoors in free stalls with an average per-cow surface area of $9 \mathrm{~m} 2$. They were fed twice daily at $08 \mathrm{~h} 00$ and $15 \mathrm{~h} 00$ with the same diet consisting of (percentage of dry mater in the diet): corn silage $(64.7 \%)$, energy concentrate $(14.8 \%)$, soybean meal (10.5\%), dried alfalfa (10\%). Cows were on twice-daily milking. Classical hygienic procedures included cleaning of teats with individual paper towel before milking and post milking teat dipping in iodine solution. The protocol was reviewed and approved by the Regional Ethics Committee for Animal Use and Care (Bretagne, France). Sampling is part of a classical veterinary practice. According to the European directive 2010/63/EU, this type of experiment does not require an authorization request.

Sampling was performed between December 2012 and March 2013, 70 days after calving on cows with parity between two and four. Animals had not developed mastitis during ongoing lactation prior to sampling: no clinical signs of mastitis and SCC values (measured twice a week on the milk collected from the four quarters, i.e., composite milk) lower than 250,000 cells/ml during 
ongoing lactation. In addition, cows retained for the study had not received any local or systemic antibiotic treatment during the ongoing lactation, considering that recent antibiotic treatment could modify microbiota. However, they had all received dry cow antibiotic therapy at the end of the previous lactation. One quarter per cow was sampled to increase biodiversity, except for four cows for which two quarters were sampled. This was done to see whether taxonomic profiles from quarters corresponding to the same animal were closest than profiles from different animals. Only quarters with SCC values lower than 100,000 cells/ml 1 week before sampling and the day of sampling were retained. In total, 31 samples corresponding to 27 animals were retained. The designations of animals are indicated by $\mathrm{V}$, followed by a number (e.g., V1, V2).

Quarters were classified based on the history of the animal and the quarter itself, taking all the lactations into account (Table S1). Briefly, quarters were classified as Healthy (Hq) when they had never encountered mastitis. These quarters correspond to animals that had no history of mastitis at all: no clinical signs of mastitis on the four quarters and SCC lower than 250,000 during all the lactations (measured twice a week on composite milk). Quarters were considered as Mastitis quarters (Mq) if they had already developed clinical mastitis (clinical signs associated to SCC increase and antibiotic treatment). All other quarters were classified as Not Determined status (NDq). These NDq quarters correspond to animals that had already undergone one or several increases of SCC (common in clinical and subclinical mastitis), but the sampled quarter has never developed clinical signs of mastitis (i.e., they are not Mq). In the case of subclinical mastitis, quarter(s) responsible for the increase of SCC is (are) generally not identified. In the case of clinical mastitis, we cannot exclude the possibility that quarters other than the Mq quarters developed subclinical mastitis concomitantly with the clinical mastitis.

\section{Sample Collection}

The sampling procedure was performed during the morning milking, essentially as previously described (Bouchard et al., 2015). Briefly, teats were thoroughly washed with osmosis water and cleaned with $70 \%$ ethanol and individual paper towels. Teat canals were then sampled in two different ways. Foremilk samples, corresponding to the milk stored in the teat cistern, were collected in sterile plastic tubes. A $5-\mathrm{mm}$ sterile Histobrush ${ }^{\circledR}$ swab (D. Dutscher, Brumath, France), was then inserted for $5 \mathrm{~mm}$ inside the teat apex and turned three times before removal. The swabs were immediately placed in tubes containing foremilk and tubes were stored on ice until processing in the laboratory.

Approximately, $30 \mathrm{ml}$ of the cisternal milk were further collected for SCC determination by LILLAB (Chateaugiron, France) and microbiological analysis: $100 \mu \mathrm{L}$ were plated on Columbia II containing 5\% sheep blood (BD, Le Pont de Claix, France) and aerobically incubated for $24-48 \mathrm{~h}$ at $37^{\circ} \mathrm{C}$. Samples were considered as infected (I) in the presence of more than five colonies corresponding to the same morphology.

Foremilk samples were processed immediately on arrival at the laboratory. Following removal of the swab, the foremilk sample was mixed with $1 / 3 \mathrm{~V}$ of sodium citrate $(1 \mathrm{M}, \mathrm{pH} 7.5)$ and centrifuged $\left(20 \mathrm{~min}, 4^{\circ} \mathrm{C}, 18,000 \mathrm{~g}\right)$. The pellet was washed in $1 \mathrm{ml}$ of sodium citrate $(20 \mathrm{~g} / \mathrm{L}, \mathrm{pH} 7.5)$, centrifuged $(15 \mathrm{~min}$, $4^{\circ} \mathrm{C}, 18,000 \mathrm{~g}$ ), treated in $100 \mu \mathrm{L}$ of $0.01 \%$ triton (Bouchard et al., 2013) and immediately resuspended in $900 \mu \mathrm{L}$ PBS $1 \mathrm{X}$ and centrifuged $\left(10 \mathrm{~min}, 4^{\circ} \mathrm{C}, 18,000 \mathrm{~g}\right)$. This last step made it possible to lyse bovine cells contained in foremilk samples and to remove released bovine DNA that could interfere with further steps due to its large quantity. The bacterial population was not altered by this step as checked during preliminary experiments by plating bacterial pellet on Plate Count Agar (Grosseron, Coueron, France) for $48 \mathrm{~h}$ at $30^{\circ} \mathrm{C}$. The pellet was then stored at $-20^{\circ} \mathrm{C}$ until DNA extraction.

\section{DNA Extraction}

Bacterial pellets were lysed for $45 \mathrm{~min}$ at $37^{\circ} \mathrm{C}$ in $360-\mu \mathrm{L}$ lysis buffer containing $20 \mathrm{mM}$ TRIS $\mathrm{HCl}(\mathrm{pH} 8), 2 \mathrm{mM}$ EDTA, $1 \%$ triton X100, $20 \mathrm{mg} / \mathrm{mL}$ Lysozyme (MP Biomedicals Illkirch, France), $50 \mathrm{U} / \mathrm{mL}$ mutanolysine (Sigma-Aldrich, Saint-Quentin Fallavier, France), and $200 \mu \mathrm{g} / \mathrm{mL}$ Lysostaphin (Sigma-Aldrich). Genomic DNA was purified using the DNeasy ${ }^{\circledR}$ Blood \& Tissue Mini Kit (Qiagen, Courtaboeuf, France), according to the manufacturer's recommendations.

\section{PCR Amplification of the V3-4 Region of Bacterial 16S rRNA Genes and Pyrosequencing of Amplicons}

PCR amplification of the V3-4 region of $16 \mathrm{~S}$ rRNA genes was done using the universal primers S-D-Bact-0341-b-S-17 and S-D-Bact-0785-a-A-21. This primer pair gave an amplicon size of $464 \mathrm{bp}$ and was identified as one of the best primer pairs for Bacteria and Archaea, giving the best overall coverage (Klindworth et al., 2013). These primers included a 10-base unique barcode to identify each sample (on forward primer only) and GS FLX Titanium Primers. The resulting composite forward primer was 5'- CCATCTCATCCCTGCGTGTCTCCGACTCAG NNNNNNNNNNCCTACGGGNGGCWGCAG-3', where the italicized sequence is the GF FLX Titanium Primer A, the NNNNNNNNNN sequence corresponds to the unique 10-base barcode, and the bold sequence is the universal primer S-D-Bact0341-b-S-17. The composite reverse primer was the same for all amplifications: 5'-CCTATCCCCTGTGTGCCTTGGCAGTCT CAGGACTACHVGGGTATCTAATCC-3' ${ }^{\prime}$, where the italicized sequence is the GF FLX Titanium Primer B and the bold sequence is the universal primer S-D-Bact-0785-a-A-21.

PCR amplification of $16 \mathrm{~S}$ rRNA was performed in duplicate using a Veriti ${ }^{\mathrm{TM}}$ 96-well thermal cycler (Applied Biosystems, Foster City, CA, USA) in a $50-\mu \mathrm{L}$ final volume containing $0.5 \mu \mathrm{M}$ forward and reverse primers, a $5 \mu \mathrm{L}$ DNA sample and $1 \times$ NEBNext High Fidelity PCR Mastermix (New England Biolabs, Evry, France). The PCR conditions were as follows: denaturation step at $95^{\circ} \mathrm{C}$ for $5 \mathrm{~min}$, followed by eight cycles of denaturation at $98^{\circ} \mathrm{C}$ for $10 \mathrm{~s}$, annealing at $61^{\circ} \mathrm{C}$ for $30 \mathrm{~s}$, and extension at $72^{\circ} \mathrm{C}$ for $30 \mathrm{~s}$ and 22 cycles of denaturation at $98^{\circ} \mathrm{C}$ for $10 \mathrm{~s}$ and extension at $72^{\circ} \mathrm{C}$ for $30 \mathrm{~s}$. A final extension step was performed for $5 \mathrm{~min}$ at $72^{\circ} \mathrm{C}$. Blank controls, in which no DNA was added to the reaction, were performed. Amplicon quality was checked on $1 \%$ agarose gel in $0.5 \mathrm{X}$ TBE. No amplicons were visible with 
blank control. Amplicons were purified with Agencourt AMPur XP magnetic beads (Beckman-Coulter) and quantified using the Quant-iT PicoGreen dsDNA Assay Kit (Invitrogen).

Pyrosequencing was performed on a GS FLX system (454/Roche) at the "Functional and Environmental Genomics" platform (OSUR, Rennes, France). DNA sequence datasets are available at the European Nucleotide Archive, under the accession number PRJEB12570.

\section{Sequence Library Analysis}

Data quality control and analyses were performed using the QIIME pipeline (Caporaso et al., 2010) hosted on the INRA MIGALE bioinformatics platform. Reads were first assigned to samples using the split_library.py script. This step also included a quality filtering step based on read length $(200<$ read length $<1000 \mathrm{bp}$ ) and quality: Number of ambiguous bases $<6$; Mean quality score $>25$; Maximum homopolymer $<$ 6; No mismatches in primers). Clustering and taxonomy assignment were then done using the pick_de_novo_otus.py script. Briefly, reads were clustered using UCLUST with a degree of similarity of $97 \%$ to generate OTUs. Taxonomy was then assigned using the Ribosomal Database Project (RDP) Classifier and the Greengenes 16S reference database (available at: http://blog.qiime.org, version available on the server in July 2014). This step generated the table of abundance for each sample (i.e., the proportion of reads corresponding to a given taxon with regard to the total number of reads of a given sample). Tables of abundance were available at different taxonomic levels: phylum (L2), class (L3), order (L4), family (L5), genus (L6).

In order to delve deeper into the taxonomic identification of discriminant genera (see below, Section Statistical Analysis), data were further analyzed by combining three taxonomy assignment methods (Blast, RDP, and Uclust) embedded in the QIIME pipeline (Caporaso et al., 2010). When the deepest RDP taxonomy assignment was at genus level, it was compared with Blast and Uclust assignment result. If Blast or Uclust assigned a deepest classification, it was retained.

Alpha diversity, which is the diversity within samples, was determined through the estimation of the Shannon index using the alpha_rarefaction.py script. The Shannon index is a widely used nonparametric index of alpha-diversity that measures richness (the total number of OTUs) and evenness (the relative abundance of OTUs). More information on the Shannon index formula can be obtained from qiime documentation (http://qiime.org/scripts/alpha_diversity.html). The Shannon index was calculated from 10 sequences onwards, with a step of 297 sequences, as automatically determined by the script.

Beta diversity, which is the diversity between samples, was estimated through the measurement of the weighted UniFrac distance, followed by Principal Component Analysis (PCA) using beta_diversity_through_plots.py script. This nontaxonomic analysis allowed to analyse diversity between samples based on the relative abundance of each OTU within the samples.

\section{Statistical Analysis}

Taxonomic profile analysis was first done by considering the abundance of the dominant genera, which means that they were present in at least 5\% abundance in a given sample. Genera present in less than $5 \%$ were also considered but they were pooled in the corresponding phylum and referred to as "phylum_others." These data, corresponding to dominant genera, are available in Table S4. A clustering of samples was then undertaken by performing a PCA on these dominant genera, followed by a hierarchical clustering using $\mathrm{R}$ software (R Development Core Team, 2013). This clustering made it possible to cluster samples into two groups.

Taxonomic profiles were then subjected to differential analysis by the use of the linear discriminant analysis (LDA) effect size (LEfSe) method (available at http://huttenhower.sph.harvard.edu/lefse/) in order to identify taxa that were discriminant between the two clusters (Segata et al., 2011). For this analysis, a table of taxa abundance including all the different taxonomic levels was used. Briefly, the first step of the LEfSe method analyzed all taxonomic units, testing whether abundance in the different clusters (designed as classes by the LEfSe method) are differentially distributed, using a Kruskal-Wallis rank sum test. An LDA model was then built to estimate the effect size of each differentially abundant taxon, i.e., to rank the differentially abundant taxa according to their relative difference among classes/clusters. This step resulted in a list of taxonomic units that are discriminative with respect to the classes/clusters and ranked according to the effect size with which they differentiate clusters.

Statistical analysis was also performed on alpha diversity (Shannon index) between groups of quarters with different health status using a Mann Whitney test with a $p<0.05$.

\section{Determination of Total Bacterial Population of Samples}

The total bacterial population of samples was estimated by quantitative PCR on 16S rRNA using primers S-D-Bact0341-b-S-17 and S-D-Bact-0785-a-A-21. Quantitative PCR was performed using a CFX96 Touch real-time system (BioRad, Hercules, CA, USA). The reaction mixture contained SsoAdvanced Universal SYBR Green Supermix (Biorad), $0.5 \mu \mathrm{M}$ of each primer and $5 \mu \mathrm{L}$ of total genomic DNA samples. Thermal cycling consisted of $30 \mathrm{~s}$ at $95^{\circ} \mathrm{C}$, followed by 40 cycles of $10 \mathrm{~s}$ at $95^{\circ} \mathrm{C}$, and $30 \mathrm{~s}$ at $60^{\circ} \mathrm{C}$. Genomic DNA of Staphylococcus aureus was used to generate standard curves. Data were normalized with regard to the volume of foremilk and are thus expressed as equivalent copy numbers of $S$. aureus genomes per $\mathrm{ml}$ of foremilk.

\section{RESULTS}

\section{Sequencing Results and Alpha Diversity Analysis}

In the present study, the teat microbiome was investigated on 31 quarters with different history of mastitis but free of inflammation at sampling time. SCC was lower than 50,000 cells/ml in all quarters retained for the analysis except one, namely V4 ARG (84,000 cells/ml). Sequencing analysis resulted in a total of 122,977 reads with an average length of $416.8 \mathrm{bp}$ 


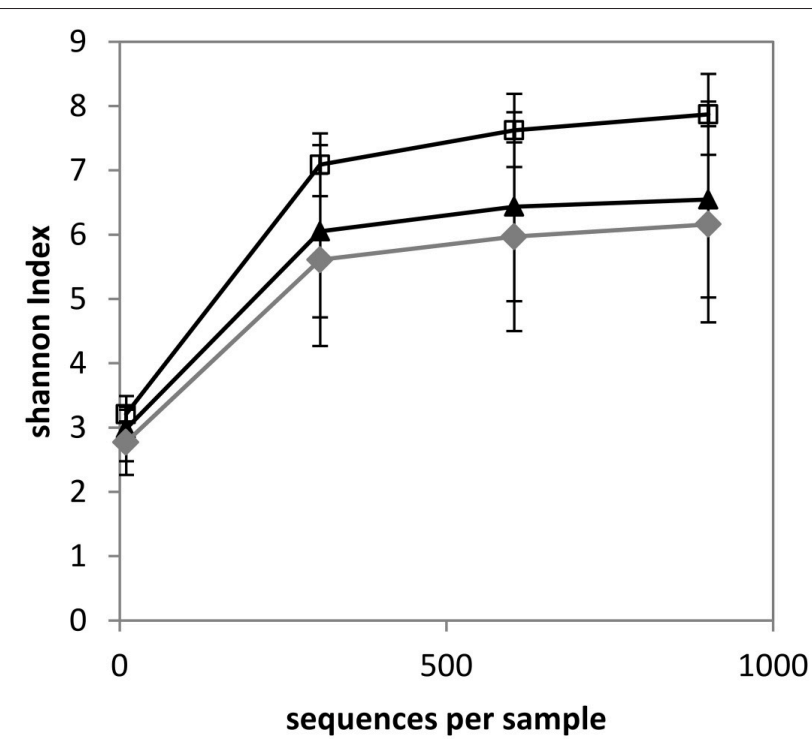

FIGURE 1 | Rarefaction curves of samples with regard to quarter status as determined by the Shannon index. White squares, healthy quarters (Hq); gray diamonds, not determined status (NDq); black triangles, quarters that have already developed mastitis (Mq).

that passed quality filters and that were assigned to bovine teat microbiome samples. Statistics regarding sequencing and filtering steps are presented in Table S2. An average number of 3967 reads per sample was obtained. Sequencing of the negative control (PCR without genomic DNA) resulted in 125 reads, which were not further analyzed.

Alpha diversity was estimated through the measurement of the Shannon index, which combines richness and evenness. Rarefaction curves were shown to flatten for each sample, indicating that sequencing was deep enough to estimate the microbiome composition (data not shown). Rarefaction curves were also determined according to quarter status (Figure 1). The Shannon index of Hq quarters was significantly higher compared to $\mathrm{Mq}$ quarters for a number of sequences per sample $\geq 307$ and it was significantly higher compared to NDq quarters for a number of sequences per sample $\geq 10$ (as determined using a Mann-Whitney Test with $p<0.05)$. As an illustration, the Shannon indices reached 7.87, 6.54, and 6.16 (for 901 sequences), for $\mathrm{Hq}, \mathrm{Mq}$, and $\mathrm{NDq}$ quarters, respectively. No significant differences were observed between $\mathrm{Mq}$ and NDq quarters.

\section{Taxonomic Profile Analysis}

Assignment of reads to taxonomic units led to the determination of taxonomic profiles of the bovine teat microbiome for quarters with different histories regarding mastitis. The relative abundance of each taxonomic unit, from phylum to genus, is presented for each sample in Table S3. Visualization of the taxonomic profile is proposed on Figure 2, which combines abundance tables at the phylum (L2) and genus (L6) levels: only dominant genera whose abundance was higher than $5 \%$ in at least one sample were included in Figure 2, whereas other genera were pooled in the corresponding phylum (see Table S4 for the corresponding abundance table combining L2 and L6 levels). An overview of Figure 2 indicates that the taxonomic profile is dominated by genera belonging to the Firmicutes, followed by genera belonging to Bacteroidetes, Actinobacteria, and to a lesser extent, Proteobacteria. Median abundances of these four phyla are 70.1, 8.1, 7.3, and 2.5\%, respectively. Taking a more in-depth look, dominant genera included Staphylococcus (with an average abundance of 23.8\%), Corynebacterium (10.1\%), Ruminococcus (4.9\%), Aerococcus (3.2\%), Bifidobacterium (2.6\%), Flacklamia (2.4\%), Jeotgalicoccus (1.3\%), Trichococcus (1.2\%), and Oscillospira (0.8\%), as well as several Lachnospiraceae such as Butyrivibrio, Dorea, and Roseburia, and two genera belonging to Bacteroidetes, namely Bacteroides and Prevotella, which all exhibited an average abundance of around $0.5 \%$. Comparison of taxonomic profiles revealed high variability between samples. Some $\mathrm{Mq}$ and $\mathrm{NDq}$ samples were clearly dominated by one genus, notably Staphylococcus and Corynebacterium, while others, notably among $\mathrm{Hq}$ and NDq quarters, showed a more balanced profile. NDq taxonomic profiles were highly variable, with a continuum of profiles ranging from some profiles resembling those of $\mathrm{Hq}$ samples to some closer to those of $\mathrm{Mq}$ samples.

Taxonomic profile analysis makes it possible to determine the relative abundance of taxonomic units but not the absolute amount of these taxonomic units within the ecosystem. In order to check whether taxonomic profile variability could be related to the variation of the total population of the microbiota, the total population was determined by performing quantitative PCR on total genomic DNA using the universal primers S-D-Bact-0341-b-S-17 and S-D-Bact-0785-a-A-21 that were used to generate our $16 \mathrm{~S}$ rRNA amplicons. Absolute quantification was performed taking the $S$. aureus genome as a standard. The median population was found to be $4.76,4.25$, and $4.6 \log _{10}$ ( $S$. aureus genome equivalent copy number) per $\mathrm{mL}$ of foremilk for $\mathrm{Mq}, \mathrm{NDq}$ and $\mathrm{Hq}$ quarters respectively, indicating no major change in the total bacterial population inside the teat.

\section{Clustering of Samples}

Comparison of bovine teat taxonomic profiles was further achieved by performing a Principal Component Analysis on the L2-L6 abundance table, followed by a hierarchical clustering (Figure 3). Good separation between $\mathrm{Hq}$ and $\mathrm{Mq}$ quarters was obtained, mainly on the first dimension, where the first two dimensions were responsible for $47 \%$ of the total variance (Figure 3A). NDq quarter distribution revealed an overlap with $\mathrm{Hq}$ and $\mathrm{Mq}$ quarter distribution. However, the centroid of the NDq quarters was closer to that of the Mq quarters. Separation between $\mathrm{Hq}$ and $\mathrm{Mq}$ quarters was also established through the hierarchical clustering. Setting the threshold for clustering at 2.6, they fell into two separate clusters, referred to as $\mathrm{C} 1$ and $\mathrm{C} 2$ on Figure 3B. Hence, all Mq quarters were clustered together into Cluster C1. This cluster also included several NDq quarters. $\mathrm{Mq}$ quarters did not cluster into a single sub-cluster within $\mathrm{C} 1$ but were instead distributed among several sub-clusters together 


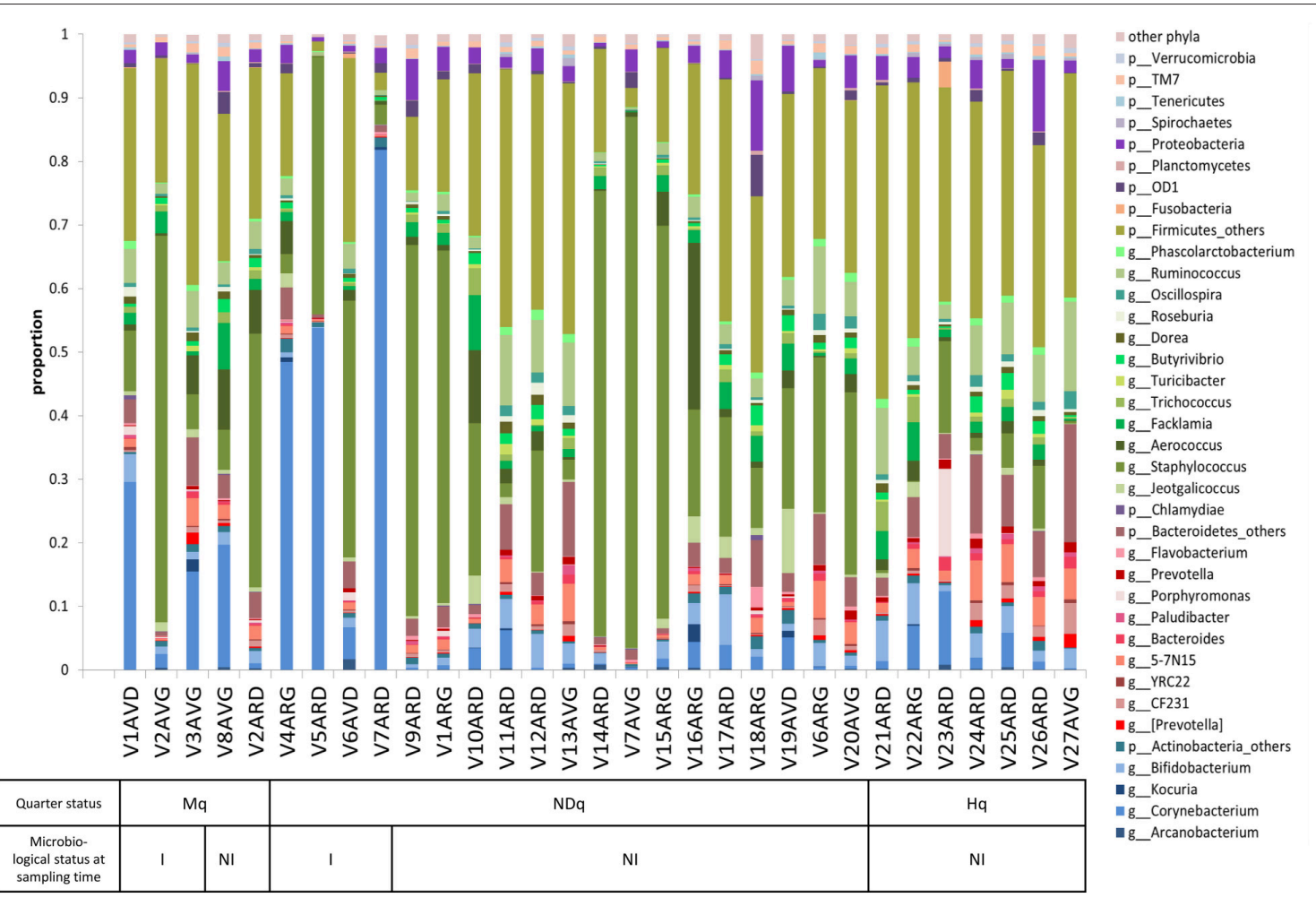

FIGURE 2 | Bovine teat taxonomic profiles combining taxonomic levels L2 (phyla) and L6 (genera). Each bar represents a sample. Genera present in at least $5 \%$ abundance in a given sample are displayed, whereas genera present in less than $5 \%$ are pooled in the corresponding phylum and referred to as "phylum_others." Actinobacteria are displayed in blue, Bacteroidetes in red, Firmicutes in green and Proteobacteria in purple. Samples were characterized with regard to: (i) quarter health status (Mq, has already developed mastitis; Hq, healthy; NDq, not determined status); and (ii) microbiological status at sampling time (I, infected; $\mathrm{NI}$, not infected), as a result of plate counts on Columbia II containing $5 \%$ sheep blood.

with NDq quarters. Similarly, all Hq quarters but one (V23ARD) were clustered together in Cluster C2, together with a few NDq quarters.

Diversity between samples was also analyzed considering OTU distribution rather than taxonomic profiles, by measuring weighted Unifrac distances between samples. As observed using taxonomic profiles, $\mathrm{Hq}$ and $\mathrm{Mq}$ quarters were separated whereas $\mathrm{NDq}$ quarters distribution revealed an overlap with $\mathrm{Hq}$ and Mq quarter distribution (Figure S1). Clusters C1 and C2, which resulted from clustering based on taxonomic profiles (see above) are indicated on Figure S1.

\section{Discriminant Analysis}

Data were subjected to discriminant analysis in order to identify differentially abundant taxonomic units between Clusters $\mathrm{C} 1$ and $\mathrm{C} 2$ (Figure 4, Table 1). Complete results of discriminant analysis, corresponding to the LDA score and $p$-value, are included in Table S5. An overview of the cladogram indicated that most of the discriminant taxa were more abundant in Cluster C2 (Figure 4A). Among the most discriminant taxa with an LDA score higher than 5, the Bacilli class was found to be more abundant in Cluster $\mathrm{C} 1$ than in Cluster C2, with a mean relative abundance of 52.6 and $22.7 \%$, respectively. This was notably related to a higher abundance of Staphylococcus, which belongs to Bacilli, and which was present at 34 and $6.7 \%$ in Clusters $\mathrm{C} 1$ and $\mathrm{C} 2$, respectively (Figure S2). Investigating in more depth the taxonomic identification of this discriminant taxon made it possible to identify $\sim 13 \%$ of total Staphylococcus reads at the species level, which mainly corresponded to $S$. aureus and S. equorum (data not shown). For two quarters, namely V1ARG and V5ARD (attributed to the C1 cluster), which were dominated by Staphylococcus, 28 and 30\% of Staphylococcus reads were assigned to $S$. aureus. For the seven other quarters (also attributed to the $\mathrm{C} 1$ cluster) that had a high abundance of Staphylococcus, reads assigned to $S$. aureus corresponded to $0.4-2.2 \%$ of Staphylococcus reads.

Conversely, the Clostridia class was the most discriminant taxonomic unit (LDA score $>5$ ), whose abundance was higher in Cluster C2 (containing healthy quarters) than in Cluster C1 (41.9 and $16.3 \%$ in C2 and C1, respectively). This was related to a higher abundance of genera belonging to the Lachnospiraceae family such as Butyrivibrio, Dorea, Roseburia, Coprococcus, Blautia and Lachnospira, and genera belonging to the Ruminococcaceae family such as Ruminococcus and Oscillospira. Among these genera, Ruminococcus was present in $\mathrm{C} 2$ and $\mathrm{C} 1$ with a mean abundance of 9.1 and $2.8 \%$, respectively. Other major discriminant taxa (LDA score > 4), whose abundance was significantly higher in Cluster C2 
TABLE 1 | Differentially abundant genera between Cluster 1 (contains Mq quarters) and Cluster 2 (contains Hq quarters) as determined by the LEfSe pipeline.

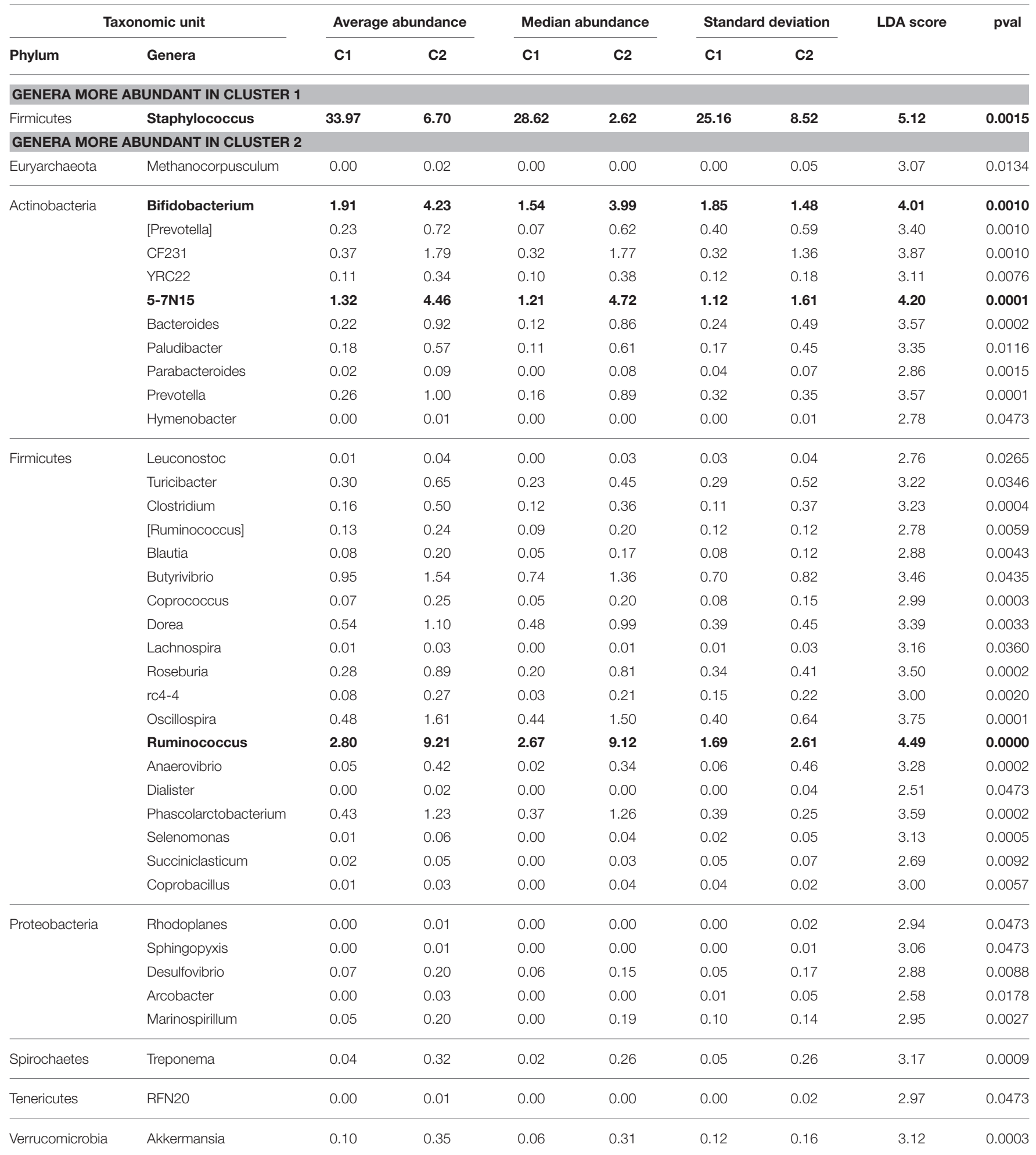

Average and median abundances (expressed as percentages) as well as standard deviation are presented for each cluster. LEfSe results include LDA score and pval. The most discriminant genera (LDA score $>4$ ) are in bold. 
A

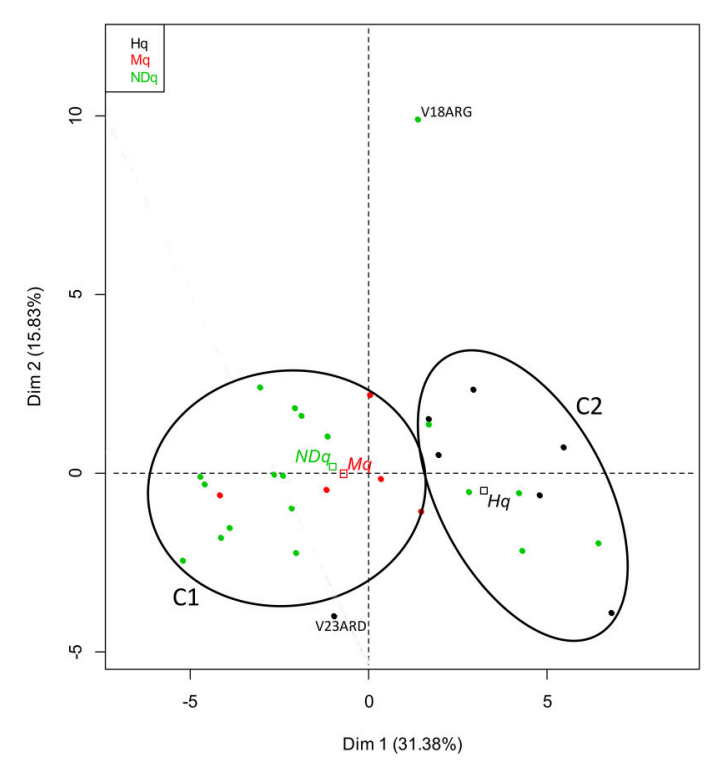

B

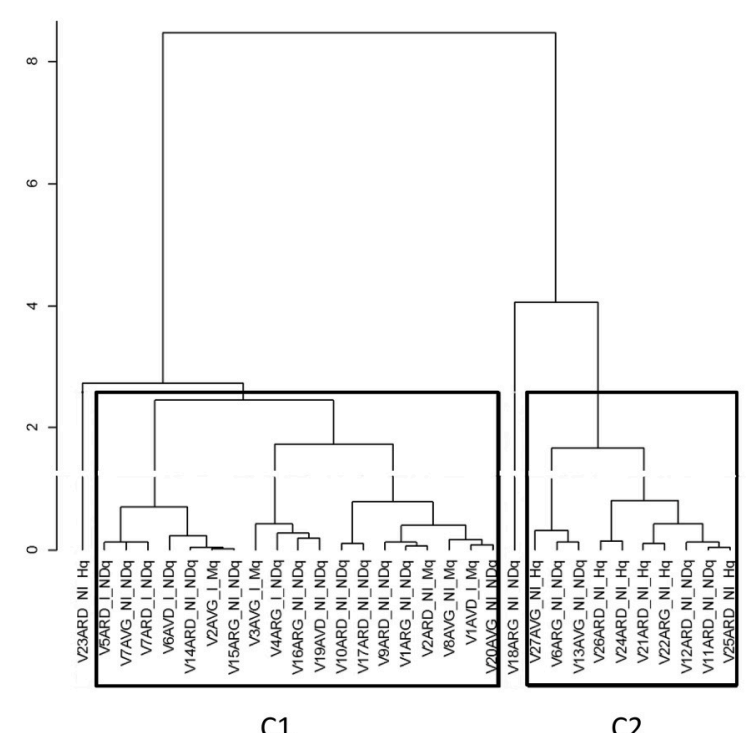

FIGURE 3 | Principal Component Analysis and hierarchical clustering on bovine teat taxonomic profiles. PCA was performed on an abundance table combining taxonomic levels L2 and L6. (A) Individual factor map. Samples are indicated by points and colored with regard to quarter health status. Centroid positions are indicated by squares for quarter status (Hq, Mq, NDq); (B) Hierarchical clustering of samples according to ACP results. All samples but one (V23ARD), classified as healthy quarters $(\mathrm{Hq})$, are clustered together (C2), whereas all samples that have already developed mastitis (Mq) and those that display an infected microbiological status (I) are clustered together (C1).

than in Cluster C1, included the Bifidobacteriales order and notably Bifidobacterium whose abundance was 1.9 and $4.2 \%$ in $\mathrm{C} 1$ and $\mathrm{C} 2$, respectively. Likewise, a higher abundance of Bacteroidetes was observed in $\mathrm{C} 2$ compared to $\mathrm{C} 1$ (relative abundance of 18.6 and $6.1 \%$ in $\mathrm{C} 2$ and $\mathrm{C} 1$, respectively). This was mainly related to a higher abundance of the Bacteroidales order. Other discriminant taxonomic units with lower potential of discrimination are listed in Table S5 and include taxa belonging to Proteobacteria, several subdominant phyla such as Cyanobacteria, Spirochaetes, Tenericutes, and Verrucomicrobia, which were more abundant in Cluster C2 than in Cluster C1, and Chlamydiae, which was more abundant in Cluster C1 than in Cluster C2.

\section{DISCUSSION}

For years now, mammary gland inflammation has been considered as the result of host pathogen interaction, a result that depends notably on bacterial and host genetic determinants (Burvenich et al., 2003; Le Marechal et al., 2011). Metagenomic exploration of several ecosystems, has revealed not only their richness and complexity but the link between their composition and ecosystem or organ functionality and health (Ravel et al., 2011; Evans et al., 2013; Belkaid and Segre, 2014; Schokker et al., 2015; Weimer, 2015). We could then wonder whether the microbiota associated with the bovine mammary gland can be related to the mammary gland health status. In this study, we investigated the composition of the bovine mammary gland microbiota in relation to quarter history with regard to mastitis using a marker gene analysis (pyrosequencing on 16S V3-V4 region). Contrary to previous studies that focus on changes in microbiome composition during mastitis (Bhatt et al., 2012; Oikonomou et al., 2012, 2014; Kuehn et al., 2013), we explored the bovine mammary gland microbiota, apart from the infectious episode, taking the animal's history rather than its health status at the time of sampling into account. In addition, contrary to these previous studies that focused on the milk microbiome, we targeted the internal teat microbiota. Infectious cycles generally start with ascending colonization of the mammary gland through the teat toward the cistern. Microbiota associated with teat canal and teat cistern epithelium may thus constitute a first microbiological barrier that can compete with pathogens. Such barrier effects were previously reported in other contexts such as gut or vaginal ecosystems (Martin, 2012; Macfarlane, 2014).

Bovine mammary microbiome analysis revealed strong variations between quarters, with some quarters clearly dominated by one taxonomic unit, whereas others displayed a more balanced profile. Of note, taxonomic profiles of quarters corresponding to the same animal were not necessarily more similar than those of quarters corresponding to different animals, as illustrated for quarters V7ARD and V7AVG, which corresponded to the same animal (V7) but did not belong to the same cluster based on their taxonomic profiles. Changes in taxonomic profiles were not related to major variations of the microbiota total population, which was similar in the three groups of quarters (Hq, Mq and NDq). Taxonomic profiles were dominated by Firmicutes in most cases, followed by three other 
A

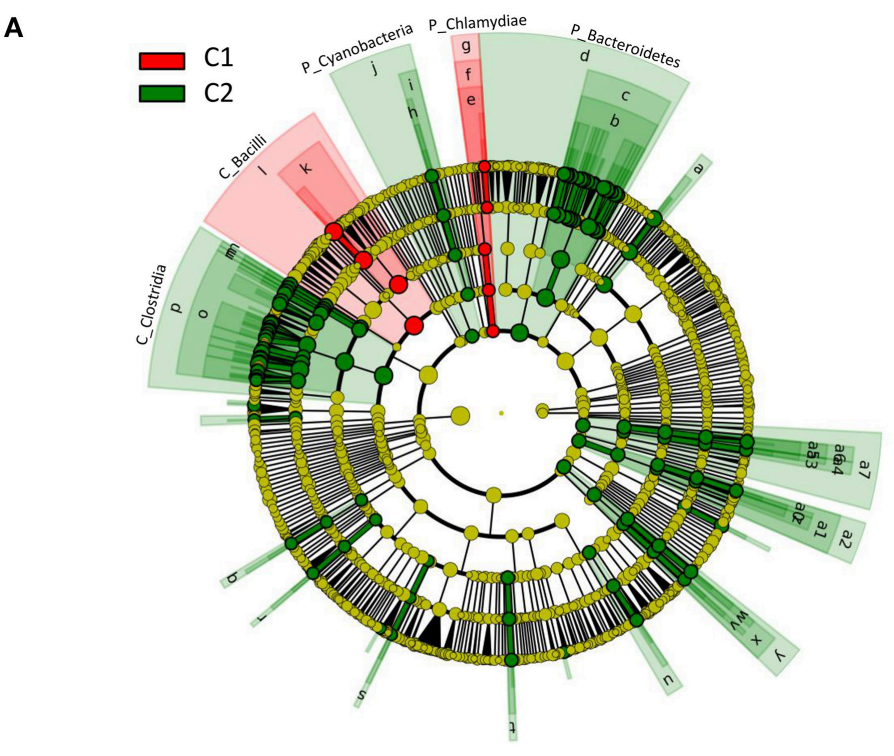

B

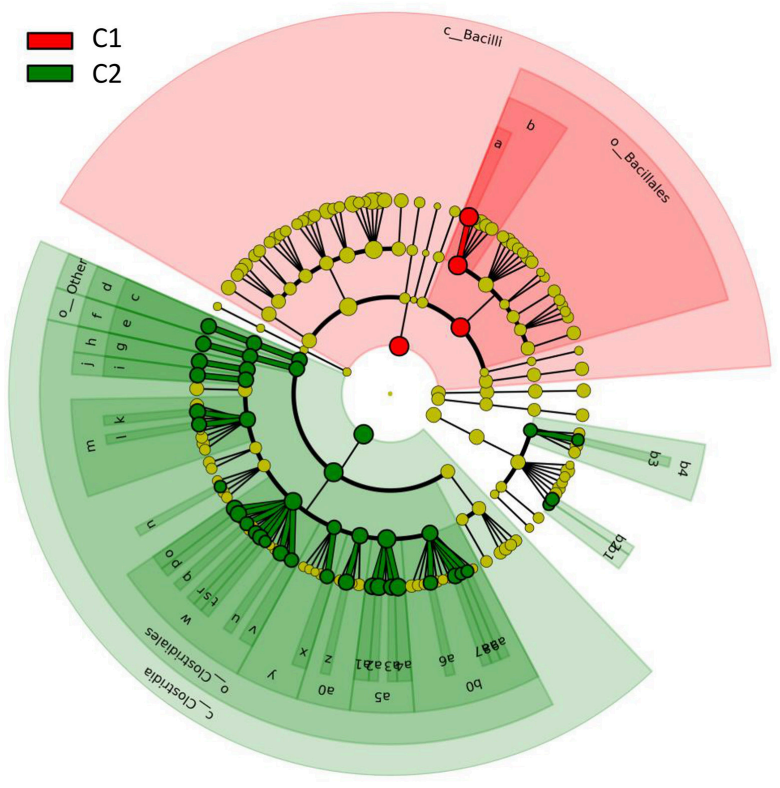

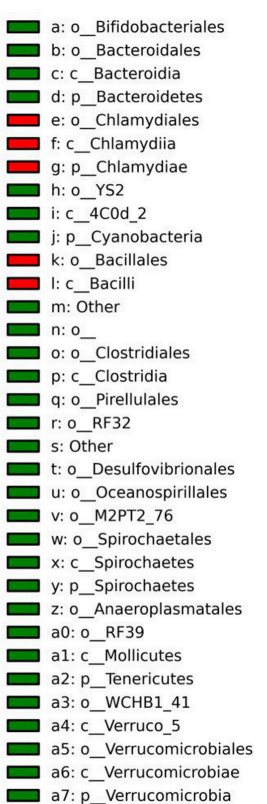

FIGURE 4 | Taxonomic representation of differentially abundant taxa between Cluster 1 (contains quarters susceptible to mastitis) and Cluster 2 (contains healthy quarters), as determined by the LEfSe pipeline. Differences are represented by the color of the cluster where the taxon is more abundant (red indicates Cluster 1 and green indicates Cluster 2). The diameter of each circle is proportional to the abundance of the taxon. (A) Root of the cladogram refers to Bacteria. Only taxonomic levels L2 (phylum) to L4 (order) are labeled. See Table S5 for taxonomic level L5 (family) and L6 (genera). (B) Detailed composition of bacteria belonging to Bacilli and Clostridia classes shown in $\mathbf{A}$ (root of the cladogram refers to the phylum Firmicutes).

phyla, namely Bacteroidetes, Actinobacteria, and Proteobacteria. Most of the dominant genera presented in Figure 2 have been described as belonging to bovine mammary microbiota, isolated from teat apices or milk, and characterized either by DGGE or 16S rRNA pyrosequencing (Braem et al., 2012, 2013; Oikonomou et al., 2012, 2014; Zhang et al., 2015). In particular, Staphylococcus is among the dominant genera in several studies on bovine milk as well as human milk (Hunt et al., 2011; Braem et al., 2012; Oikonomou et al., 2014). Nevertheless, some discrepancies exist since the relative abundance of these genera varies between the different studies. For instance, Propionibacterium and Aeribacillus were the dominant genera in healthy quarters of Oikonomou's study $(\sim 10 \%$ average abundance; Oikonomou et al., 2012), whereas they were poorly present in our study 
(average abundance lower than 0.5\%). Likewise, Streptococcus was among the most prevalent genera, regardless of the quarter status in Oikonomou's report (Oikonomou et al., 2014), whereas Streptococcus mean abundance was below $0.04 \%$ in our study. Several explanations may account for such discrepancies, including the sampling itself and technical parameters such as the method of bacterial lysis and genomic DNA extraction and the 16S rRNA variable region retained for amplification. Of note, DNA extraction was performed on a bacterial pellet washed and treated by triton to lyse bovine cells and remove contaminating bovine DNA. We cannot totally exclude that these separation steps led to the elimination or killing of some bacterial species, although the total bacterial population was not altered. The impact of DNA extraction methods and sampling techniques has been clearly demonstrated on the microbial community composition of cow and sheep rumen (Henderson et al., 2013). In addition, microbiota has already been found to vary between herd and geographical areas (Espeche et al., 2012). Data analysis can also introduce some bias. For instance, in this study, sequences have not been checked for chimeras. Chimeras have been shown to have minor influence on results generated by 454 pyrosequencing (removing of less than $1 \%$ of reads), whereas denoising pipelines such as ChimeraSlayer can introduce changes to the reads, thus questioning the relevance of these denoising pipelines (Gaspar and Thomas, 2013).

\section{Variations of the Bovine Teat Microbiome Correlated with Mastitis History}

Firstly, analysis of taxonomic profile diversity revealed that alpha diversity significantly varied with regard to the quarter's status. Healthy quarters exhibited a significantly higher diversity than quarters that had already undergone clinical mastitis, as revealed by the Shannon diversity index of 7.87 and 6.54 for $\mathrm{Hq}$ and $\mathrm{Mq}$ quarters, respectively. Such changes of diversity in relation to health status have already been observed by Braem et al. (2012), who reported a higher number of genera in the teat apex from non-infected quarters, as determined by DGGE. Likewise, a lower diversity has been reported in the uterine microbiota of cows suffering from metritis compared to healthy animals, as revealed by DNA pyrosequencing (Santos and Bicalho, 2012).

Secondly, clustering of taxonomic profiles made it possible to separate all heathy quarters but one (V23ARD) from those that had already undergone clinical mastitis. Of note, analysis of diversity between samples based on OTU distribution was in agreement with clustering obtained using taxonomic profiles. In particular, separation between $\mathrm{Hq}$ and $\mathrm{Mq}$ quarters was obtained. The sample V23ARD was more closely related to the other $\mathrm{Hq}$ quarters when considering OTU distribution. It should be noticed here that, in the lack of SCC recording at quarter level on the previous lactations, assignment of quarters to Hq was based on SCC recording on composite milk. However, we could not totally exclude, by using a threshold lower than $250,000 \mathrm{cell} / \mathrm{mL}$ on composite milk, that some Hq quarters had previously developed subclinical mastitis. Indeed, in a previously published meta-analysis, the mean SCC was $68,000 \mathrm{cells} / \mathrm{ml}$ in bacteriologically negative quarters, whereas it was higher than 105,000 cells $/ \mathrm{ml}$ in quarters that harbored intra-mammary infections (Djabri et al., 2002). The likehood that cows with composite milk SCC between 100,000 and 250,000 cells/mL had at least one inflamed quarter was not negligible. Nevertheless, despite the above-mentioned reservations on our criteria, almost all $\mathrm{Hq}$ quarters clustered together and were separated from $\mathrm{Mq}$ quarters.

One $\mathrm{Hq}$ quarter was isolated, namely V23ARD, which contained high abundances of Porphyromonas and Fusobacteria compared to all the other samples (Figure 2). Porphyromonas levii and Fusobacterium necrophorum have been detected in most of the mastitic milk samples by Oikonomou and coworkers, although they were not directly responsible for the mastitis (Oikonomou et al., 2012). Both species, F. necrophorum and $P$. levii, have been shown to be involved in summer mastitis, acting in synergy with other pathogens such as Trueperella pyogenes (Pyörälä et al., 1992). Interestingly, this animal (V23) exhibited a moderate increase of SCC on composite milk ( $>300,000$ cells $/ \mathrm{mL}$ on milk collected from the four quarters) at the end of the analyzed lactation. Whether this SCC increase was related to a subclinical mastitis on this quarter at the end of lactation was however not determined.

NDq quarters clustered either with Mq quarters in Cluster $\mathrm{C} 1$ or $\mathrm{Hq}$ quarters in Cluster C2. The clustering of most NDq quarters with $\mathrm{Mq}$ quarters strongly suggests that several NDq quarters, but not all, have a history of subclinical mastitis. Of note, all NDq quarters that were infected but not inflamed at the time of sampling were included in $\mathrm{C} 1$. However, we cannot totally exclude that the presence of pathogens in cistern milk sample was due to contamination by pathogens present in the teat cistern or teat canal and washed out during milking.

Variation of the taxonomic profile in relation to heath status has already been reported in the mammary gland and uterine contexts (Santos et al., 2011; Braem et al., 2012; Oikonomou et al., 2012, 2014; Santos and Bicalho, 2012; Kuehn et al., 2013). However, all these studies focused on changes in the bacterial community at the time of inflammation. Here, we showed that the microbiota composition of quarters with different health statuses was also altered apart from the infectious episode (of note, animals had not received antibiotic treatment for the last 2 months at least). These different taxonomic profiles may result from an infectious episode and or antibiotic treatment. Alternatively, we cannot exclude that taxonomic profiles were already altered prior to the infection and that these alterations contributed to the infection.

\section{Discriminant Analysis Revealed Taxonomic Markers of a Quarter's Health Status}

Discriminant analysis between Cluster $\mathrm{C}$, which includes all Mq quarters, and Cluster $\mathrm{C} 2$, which includes all $\mathrm{Hq}$ quarters but one, led to the identification of taxonomic markers of these two clusters. Discrimination occurred at different taxonomic levels. Most taxonomic markers belong to Firmicutes and exhibited a higher abundance in C2. The most discriminant taxa were also among the most abundant ones. Hence, within Firmicutes, Bacilli were significantly more abundant in $\mathrm{C} 1$ than in $\mathrm{C} 2$, with average 
abundance of 52.6 and $22.7 \%$, respectively, whereas Clostridia were significantly more abundant in $\mathrm{C} 2$ than in $\mathrm{C} 1$, with average abundances of 41.9 and $16.3 \%$ in C2 and C1, respectively. Other discriminant taxa included Bifidobacterium and the phylum Bacteroidetes, whose average abundances were 2-fold and 3-fold higher in $\mathrm{C} 2$ quarters than in $\mathrm{C} 1$, respectively. The origin of these differential taxonomic profiles and, notably, the change in the Bacilli/Clostridia ratio remain to be elucidated. Several of the genera belonging to Clostridia as well as to Bacteroidetes are common with the rumen microbiome (Jami and Mizrahi, 2012; McCann et al., 2014; Sandri et al., 2014; Kumar et al., 2015), suggesting a transfer between the rumen to the mammary gland, possibly in a way similar to the gut-breast axis observed from mother to neonate in humans (Jost et al., 2014) but more probably by ascending colonization through the teat canal (fecal contamination).

The high abundance of Bacilli in C1 is notably related to a high amount of Staphylococcus, whose abundance was $34 \%$ on average in $\mathrm{C} 1$ (vs. $6.7 \%$ in $\mathrm{C} 2$ ) and even reached $83 \%$ in the quarter V7AVG. Two of the nine quarters dominated by Staphylococcus, namely V1ARG and V5ARD, had $\sim 30 \%$ of Staphylococcus reads assigned to $S$. aureus. The seven other quarters had only $0.4-$ $2.2 \%$ of Staphylococcus reads assigned to S. aureus, indicating that other and possibly multiple Staphylococcus species were responsible for this profile. $S$. aureus and coagulase negative staphylococci (CNS) are among the main etiologic agents involved in mastitis (Dufour et al., 2012; Keane et al., 2013, p. 2). S. aureus is considered as a major pathogen in bovine mastitis, together with Streptococcus uberis and Escherichia coli, while CNS are considered as minor pathogens, leading to milder inflammations and subclinical mastitis. The Staphylococci reservoir is mainly the mammary gland, whereas E. coli is considered as an environmental pathogen. The $S$. uberis reservoir includes the environment and the mammary gland. E. coli generally rapidly leads to acute infections followed by resolution, while $S$. aureus and $S$. uberis are likely to persist within the mammary gland. In agreement, we found Staphylococci in all quarters, whereas E. coli was not detected and the Streptococcus sp. mean abundance was $0.04 \%$. Although, it may be speculative since we do not know which pathogen was involved in the recorded SCC increases, the higher abundance of Staphylococci in $\mathrm{C} 1$ quarters may have favored their ability to reach the cistern through ascending colonization, thus provoking inflammation. It may also have favored the emergence of other pathogens through the interaction with the immune system. Such "collaboration" between pathogens has already been observed, i.e., in the bovine genital tract context. Hence, metritis often starts by contamination of the uterine lumen by $E$. coli, followed by postinfection by other species such as Arcanobacterium pyogenes or Fusobacterium necrophorum (Williams et al., 2007). Interestingly, Corynebacterium has also been previously associated with intramammary infections, but as a colonizer of the teat canal rather than a causal agent of mastitis (Braem et al., 2012). Sam Ma et al. (2015) have recently suggested a potential 'evil' alliance of Staphylococcus and Corynebacterium in the human milk microbiome against the benign microbiota, leading to dysbiosis and enabling mastitis (Sam Ma et al., 2015). Corynebacterium was not discriminant between $\mathrm{C} 1$ and $\mathrm{C} 2$ due to strong variations of its abundance between quarters. However, six quarters belonging to C1, namely V1AVD, V3AVG, V4ARG, V5ARD, V7ARD, and V8AVG, exhibited a high abundance of Corynebacterium, from 15 to $82 \%$, whereas the mean abundance of Corynebacterium in C2 quarters was much lower at $2.4 \%$.

\section{CONCLUDING REMARKS}

In this study, we showed that the bovine teat bacterial community varies in relation to animal history regarding mastitis, apart from the infectious episode. Hence, healthy quarters and those that had already developed mastitis exhibited divergent taxonomic profiles that could be separated into two clusters. Discriminant analysis made it possible to identify taxonomic markers of these two clusters, which could become good candidates to develop diagnostic tools of mammary gland health. However, in this study, the exploration of bovine mammary microbiota was done on one herd. Extending this work to other herds will help to test the robustness of these taxonomic markers, to adjust them or define new ones. Several parameters probably influence microbiota, and should be investigated, including diet, herd management, animal housing, milking number, hygienic procedures during milking, or animal genetics and lactation number. Of note, the average number of lactations was not statistically different in clusters C1 and C2 (2.63 and 2.5 in C1 and $\mathrm{C} 2$ respectively) nor in quarter groups (average number of lactations of 2.60, 2.55 and 2.62 for Mq, NDq, and Hq quarters respectively). However, only multiparous animals were included in the study, mainly in the second and third lactations.

The existence of differential taxonomic profiles between C2 and $\mathrm{C} 1$ questions whether mammary gland microbiota could interfere with host pathogen interaction. However, investigating the dynamics of this bacterial community all along the lactations, starting on animals prior to any mammary gland infection, will be necessary to determine whether differences of the bacterial community in $\mathrm{C} 1$ contributes to or results from mastitis development, or both. Additional investigations will also be necessary to investigate the functionality of this microbiota: are these bacteria alive? How do they interact with mammary gland epithelium? At the very least, these results encourage us to take the teat microbiota into account in bovine mammary gland health management and to explore strategies that would preserve or restore a balanced microbiota as a prophylactic measure.

\section{AUTHOR CONTRIBUTIONS}

Substantial contributions to the conception or design of the work: SE, YL, PM; acquisition: LR, DB, JL, PL, JA, SE; analysis and interpretation of data for the study: SE, HF, AN. Drafting of the study: SE, HF; revising it critically for important intellectual content: all of the authors. All of the authors approved the final version of the manuscript and agreed to be accountable for all aspects of the work by ensuring that questions related to the accuracy or integrity of any part of the work are appropriately investigated and resolved. 


\section{FUNDING}

This work was financially supported by the INRA-funded MEM project (Milk Ecosystem and Udder Health).

\section{ACKNOWLEDGMENTS}

We are grateful to the Biogenouest Genomics and the Environmental and Functional Genomics Platform core facility for technical supports. We are grateful to the INRA MIGALE bioinformatics platform (http://migale.jouy.inra.fr) for providing computational resources. We thank Antoine Eveno, Michel Fargetton, Arnaud Mottin, Joseph Orinel and Jean Parois for their technical assistance during the sampling campaign. We thank Gail Wagman for revising the English.

\section{SUPPLEMENTARY MATERIAL}

The Supplementary Material for this article can be found online at: http://journal.frontiersin.org/article/10.3389/fmicb. 2016.00480

Figure S1 | Principal Component Analysis based on weighted UniFrac distances. Samples are indicated by points and colored with regard to quarter health status: Hq, (blue); Mq, (red); NDq, (green).

Figure S2 | Selected boxplot of taxonomic units that are differentially abundant between Cluster 1 (contains quarters susceptible to mastitis) and Cluster 2 (contains healthy quarters) as determined by the LEfSe

\section{REFERENCES}

Belkaid, Y., and Segre, J. A. (2014). Dialogue between skin microbiota and immunity. Science 346, 954-959. doi: 10.1126/science.12 60144

Bhatt, V. D., Ahir, V. B., Koringa, P. G., Jakhesara, S. J., Rank, D. N., Nauriyal, D. S., et al. (2012). Milk microbiome signatures of subclinical mastitis-affected cattle analysed by shotgun sequencing. J. Appl. Microbiol. 112, 639-650. doi: 10.1111/j.1365-2672.2012.05 244.x

Bouchard, D. S., Rault, L., Berkova, N., Le Loir, Y., and Even, S. (2013). Inhibition of Staphylococcus aureus invasion into bovine mammary epithelial cells by contact with live Lactobacillus casei. Appl. Environ. Microbiol. 79, 877-885. doi: 10.1128/AEM.03323-12

Bouchard, D. S., Seridan, B., Saraoui, T., Rault, L., Germon, P., Gonzalez-Moreno, C., et al. (2015). Lactic acid bacteria isolated from bovine mammary microbiota: potential allies against bovine mastitis. PLoS ONE 10:e0144831. doi: 10.1371/journal.pone.01 44831

Braem, G., De Vliegher, S., Verbist, B., Heyndrickx, M., Leroy, F., and De Vuyst, L. (2012). Culture-independent exploration of the teat apex microbiota of dairy cows reveals a wide bacterial species diversity. Vet. Microbiol. 157, 383-390. doi: 10.1016/j.vetmic.2011.12.031

Braem, G., De Vliegher, S., Verbist, B., Piessens, V., Van Coillie, E., De Vuyst, L., et al. (2013). Unraveling the microbiota of teat apices of clinically healthy lactating dairy cows, with special emphasis on coagulasenegative staphylococci. J. Dairy Sci. 96, 1499-1510. doi: 10.3168/jds.20 12-5493

Burvenich, C., Van Merris, V., Mehrzad, J., Diez-Fraile, A., and Duchateau, L. (2003). Severity of E. coli mastitis is mainly determined by cow factors. Vet. Res. 34, 521-564. doi: 10.1051/vetres:20 03023 pipeline. The box represents the 75\% (upper) and 25\% (lower) quartiles and the black line inside the box represents the median. Abundance of the classes Bacilli (A) and Clostridia (B) the phylum Bacteroidetes (C) and the genera Staphylococcus (D) Ruminococcus (E) and Bifidobacterium (F).

Table S1 | Quarter status based on the history of the animal.

Table S2 | Sequencing overview and filtering statistics. Reads were filtered with regard to their length and quality and assigned to samples using the split_library.py script of QIIME.

Table S3 | Bovine teat taxonomic profiles combining all taxonomic levels (from phylum to genera). The abundance of each taxonomic unit is presented for each sample, completed by the average and median abundances for each cluster (columns AN-AS). Samples were characterized with regard to: (i) quarter health status (Mq, has already developed mastitis; Hq, healthy (no mastitis at all); NDq, unknown status), and (ii) microbiological status at sampling time (l, infected; $\mathrm{NI}$, not infected).

Table S4 | Bovine teat taxonomic profiles combining taxonomic levels L2 (phyla) and L6 (genera). Genera present in at least 5\% abundance in a given sample are displayed, whereas genera present in less than $5 \%$ are pooled in the corresponding phylum and referred to as "phylum_others." Samples were characterized with regard to (i) quarter health status (Mq, has already developed mastitis; Hq, healthy (no mastitis at all); NDq, unknown status), and (ii) microbiological status at sampling time (I, infected; NI, not infected). The SCC of the quarter at the time of sampling is also included.

Table S5 | Differentially abundant taxonomic units between Cluster 1 (contains Mq quarters) and Cluster 2 (contains Hq quarters) as determined by the LEfSe pipeline. Average and median abundances (expressed as percentages) as well as standard deviation are presented for each cluster. LEfSe results include LDA score and pval.

Caporaso, J. G., Kuczynski, J., Stombaugh, J., Bittinger, K., Bushman, F. D., Costello, E. K., et al. (2010). QIIME allows analysis of highthroughput community sequencing data. Nat. Methods 7, 335-336. doi: 10.1038/nmeth.f.303

Djabri, B., Bareille, N., Beaudeau, F., and Seegers, H. (2002). Quarter milk somatic cell count in infected dairy cows: a meta-analysis. Vet. Res. 33, 335-357. doi: 10.1051/vetres:2002021

Dufour, S., Dohoo, I. R., Barkema, H. W., Descôteaux, L., Devries, T. J., Reyher, K. K., et al. (2012). Epidemiology of coagulase-negative staphylococci intramammary infection in dairy cattle and the effect of bacteriological culture misclassification. J. Dairy Sci. 95, 3110-3124. doi: 10.3168/jds.20 11-5164

Espeche, M. C., Pellegrino, M., Frola, I., Larriestra, A., Bogni, C., and NaderMacías, M. E. (2012). Lactic acid bacteria from raw milk as potentially beneficial strains to prevent bovine mastitis. Anaerobe 18, 103-109. doi: 10.1016/j.anaerobe.2012.01.002

Evans, J. M., Morris, L. S., and Marchesi, J. R. (2013). The gut microbiome: the role of a virtual organ in the endocrinology of the host. J. Endocrinol. 218, R37-R47. doi: 10.1530/JOE-13-0131

Gaspar, J. M., and Thomas, W. K. (2013). Assessing the consequences of denoising marker-based metagenomic data. PLoS ONE 8:e60458. doi: 10.1371/journal.pone.0060458

Heikkilä, A. M., Nousiainen, J. I., and Pyörälä, S. (2012). Costs of clinical mastitis with special reference to premature culling. J. Dairy Sci. 95, 139-150. doi: 10.3168/jds.2011-4321

Henderson, G., Cox, F., Ganesh, S., Jonker, A., Young, W., Global Rumen Census Collaborators, et al. (2015). Rumen microbial community composition varies with diet and host, but a core microbiome is found across a wide geographical range. Sci. Rep. 5:14567. doi: 10.1038/srep 14567

Henderson, G., Cox, F., Kittelmann, S., Miri, V. H., Zethof, M., Noel, S. J., et al. (2013). Effect of DNA extraction methods and sampling techniques on the 
apparent structure of cow and sheep rumen microbial communities. PLoS ONE 8:e74787. doi: 10.1371/journal.pone.0074787

Hunt, K. M., Foster, J. A., Forney, L. J., Schütte, U. M. E., Beck, D. L., Abdo, Z., et al. (2011). Characterization of the diversity and temporal stability of bacterial communities in human milk. PLOS ONE 6:e21313. doi: 10.1371/journal.pone.0021313

Jami, E., and Mizrahi, I. (2012). Composition and similarity of bovine rumen microbiota across individual animals. PLOS ONE 7:e33306. doi: 10.1371/journal.pone.0033306

Jami, E., White, B. A., and Mizrahi, I. (2014). Potential role of the bovine rumen microbiome in modulating milk composition and feed efficiency. PLoS ONE 9:e85423. doi: 10.1371/journal.pone.00 85423

Jewell, K. A., McCormick, C. A., Odt, C. L., Weimer, P. J., and Suen, G. (2015). Ruminal bacterial community composition in dairy cows is dynamic over the course of two lactations and correlates with feed efficiency. Appl. Environ. Microbiol. 81, 4697-4710. doi: 10.1128/AEM.007 20-15

Jost, T., Lacroix, C., Braegger, C. P., Rochat, F., and Chassard, C. (2014). Vertical mother-neonate transfer of maternal gut bacteria via breastfeeding. Environ. Microbiol. 16, 2891-2904. doi: 10.1111/1462-2920. 12238

Keane, O. M., Budd, K. E., Flynn, J., and McCoy, F. (2013). Pathogen profile of clinical mastitis in Irish milk-recording herds reveals a complex aetiology. Vet. Rec. 173, 17. doi: 10.1136/vr.101308

Kim, H. B., and Isaacson, R. E. (2015). The pig gut microbial diversity: understanding the pig gut microbial ecology through the next generation high throughput sequencing. Vet. Microbiol. 177, 242-251. doi: 10.1016/j.vetmic.2015.03.014

Klindworth, A., Pruesse, E., Schweer, T., Peplies, J., Quast, C., Horn, M., et al. (2013). Evaluation of general 16S ribosomal RNA gene PCR primers for classical and next-generation sequencing-based diversity studies. Nucleic Acids Res. 41, el. doi: 10.1093/nar/gks808

Knudsen, L. R. V., Karstrup, C. C., Pedersen, H. G., Agerholm, J. S., Jensen, T. K., and Klitgaard, K. (2014). Revisiting bovine pyometra-New insights into the disease using a culture-independent deep sequencing approach. Vet. Microbiol. 175, 319-324. doi: 10.1016/j.vetmic.2014.12.006

Kuehn, J. S., Gorden, P. J., Munro, D., Rong, R., Dong, Q., Plummer, P. J., et al. (2013). Bacterial community profiling of milk samples as a means to understand culture-negative bovine clinical mastitis. PLoS ONE 8:e61959. doi: 10.1371/journal.pone.0061959

Kumar, S., Indugu, N., Vecchiarelli, B., and Pitta, D. W. (2015). Associative patterns among anaerobic fungi, methanogenic archaea, and bacterial communities in response to changes in diet and age in the rumen of dairy cows. Front. Microbiol. 6:781. doi: 10.3389/fmicb.2015. 00781

Le Marechal, C., Jardin, J., Jan, G., Even, S., Pulido, C., Guibert, J. M., et al. (2011). Staphylococcus aureus seroproteomes discriminate ruminant isolates causing mild or severe mastitis. Vet. Res. 42:35. doi: 10.1186/1297-971 6-42-35

Macfarlane, S. (2014). Antibiotic treatments and microbes in the gut. Environ. Microbiol. 16, 919-924. doi: 10.1111/1462-2920. 12399

Machado, V. S., Oikonomou, G., Bicalho, M. L. S., Knauer, W. A., Gilbert, R., and Bicalho, R. C. (2012). Investigation of postpartum dairy cows' uterine microbial diversity using metagenomic pyrosequencing of the 16S rRNA gene. Vet. Microbiol. 159, 460-469. doi: 10.1016/j.vetmic.2012.04.033

Martin, D. H. (2012). The microbiota of the vagina and its influence on women's health and disease. Am. J. Med. Sci. 343, 2-9. doi: 10.1097/MAJ.0b013e31823ea228

McCann, J. C., Wickersham, T. A., and Loor, J. J. (2014). High-throughput methods redefine the rumen microbiome and its relationship with nutrition and metabolism. Bioinforma. Biol. Insights 8, 109-125. doi: 10.4137/BBI.S15389

Minuti, A., Palladino, A., Alqarni, S., Agrawal, A., Piccioli-Capelli, F., Hidalgo, F., et al. (2015). Abundance of ruminal bacteria, epithelial gene expression, and systemic biomarkers of metabolism and inflammation are altered during the peripartal period in dairy cows. J. Dairy Sci. 98, 8940-8951. doi: 10.3168/jds.201 5-9722
Mohammed, R., Brink, G. E., Stevenson, D. M., Neumann, A. P., Beauchemin, K. A., Suen, G., et al. (2014). Bacterial communities in the rumen of Holstein heifers differ when fed orchardgrass as pasture vs. hay. Front. Microbiol. 5:689. doi: $10.3389 /$ fmicb. 2014.00689

Myer, P. R., Smith, T. P. L., Wells, J. E., Kuehn, L. A., and Freetly, H. C. (2015). Rumen microbiome from steers differing in feed efficiency. PLoS ONE 10:e0129174. doi: 10.1371/journal.pone.0129174

Nader-Macías, M. E., Otero, M. C., Espeche, M. C., and Maldonado, N. C. (2008). Advances in the design of probiotic products for the prevention of major diseases in dairy cattle. J. Ind. Microbiol. Biotechnol. 35, 1387-1395. doi: 10.1007/s10295-008-0438-2

Oikonomou, G., Bicalho, M. L., Meira, E., Rossi, R. E., Foditsch, C., Machado, V. S., et al. (2014). Microbiota of cow's milk; distinguishing healthy, sub-clinically and clinically diseased quarters. PLoS ONE 9:e85904. doi: 10.1371/journal.pone.0085904

Oikonomou, G., Machado, V. S., Santisteban, C., Schukken, Y. H., and Bicalho, R. C. (2012). Microbial diversity of bovine mastitic milk as described by pyrosequencing of metagenomic 16s rDNA. PLoS ONE 7:e47671. doi: 10.1371/journal.pone.0047671

Pyörälä, S., Jousimies-Somer, H., and Mero, M. (1992). Clinical, bacteriological and therapeutic aspects of bovine mastitis caused by aerobic and anaerobic pathogens. Br. Vet. J. 148, 54-62. doi: 10.1016/0007-1935(92)9 0067-B

R Development Core Team (2013). R: A Language and Environment for Statistical Computing. Vienna: R Foundation for Statistical. Available online at: http://www.R-project.org

Ravel, J., Gajer, P., Abdo, Z., Schneider, G. M., Koenig, S. S. K., McCulle, S. L., et al. (2011). Vaginal microbiome of reproductive-age women. Proc. Natl. Acad. Sci. U.S.A. 108 (Suppl. 1), 4680-4687. doi: 10.1073/pnas.10026 11107

Rodrigues, N. F., Kästle, J., Coutinho, T. J. D., Amorim, A. T., Campos, G. B., Santos, V. M., et al. (2015). Qualitative analysis of the vaginal microbiota of healthy cattle and cattle with genital-tract disease. Genet. Mol. Res. GMR 14, 6518-6528. doi: 10.4238/2015.June.12.4

Sam Ma, Z., Guan, Q., Ye, C., Zhang, C., Foster, J. A., and Forney, L. J. (2015). Network analysis suggests a potentially "evil" alliance of opportunistic pathogens inhibited by a cooperative network in human milk bacterial communities. Sci. Rep. 5:8275. doi: 10.1038/srep 08275

Sandri, M., Manfrin, C., Pallavicini, A., and Stefanon, B. (2014). Microbial biodiversity of the liquid fraction of rumen content from lactating cows. Anim. Int. J. Anim. Biosci. 8, 572-579. doi: 10.1017/S17517311140 00056

Santos, T. M., and Bicalho, R. C. (2012). Diversity and succession of bacterial communities in the uterine fluid of postpartum metritic, endometritic and healthy dairy cows. PLOS ONE 7:e53048. doi: 10.1371/journal.pone.00 53048

Santos, T. M., Gilbert, R. O., and Bicalho, R. C. (2011). Metagenomic analysis of the uterine bacterial microbiota in healthy and metritic postpartum dairy cows. J. Dairy Sci. 94, 291-302. doi: 10.3168/jds.201 $0-3668$

Schokker, D., Veninga, G., Vastenhouw, S. A., Bossers, A., de Bree, F. M., Kaal-Lansbergen, L. M. T. E., et al. (2015). Early life microbial colonization of the gut and intestinal development differ between genetically divergent broiler lines. BMC Genomics 16, 418. doi: 10.1186/s12864-0151646-6

Segata, N., Izard, J., Waldron, L., Gevers, D., Miropolsky, L., Garrett, W. S., et al. (2011). Metagenomic biomarker discovery and explanation. Genome Biol. 12:R60. doi: 10.1186/gb-2011-12-6-r60

Vayssier-Taussat, M., Albina, E., Citti, C., Cosson, J.-F., Jacques, M.-A., Lebrun, M.-H., et al. (2014). Shifting the paradigm from pathogens to pathobiome: new concepts in the light of meta-omics. Front. Cell. Infect. Microbiol. 4:29. doi: 10.3389/fcimb.2014.00029

Veneman, J. B., Muetzel, S., Hart, K. J., Faulkner, C. L., Moorby, J. M., Perdok, H. B., et al. (2015). Does dietary mitigation of enteric methane production affect rumen function and animal productivity in dairy cows? PLoS ONE 10:e0140282. doi: 10.1371/journal.pone.01 40282 
Weimer, P. J. (2015). Redundancy, resilience, and host specificity of the ruminal microbiota: implications for engineering improved ruminal fermentations. Front. Microbiol. 6:296. doi: 10.3389/fmicb.2015.00296

Williams, E. J., Fischer, D. P., Noakes, D. E., England, G. C., Rycroft, A., Dobson, H., et al. (2007). The relationship between uterine pathogen growth density and ovarian function in the postpartum dairy cow. Theriogenology $68,549-559$. doi: 10.1016/j.theriogenology.2007.04.056

Zhang, R., Huo, W., Zhu, W., and Mao, S. (2015). Characterization of bacterial community of raw milk from dairy cows during subacute ruminal acidosis challenge by high-throughput sequencing. J. Sci. Food Agric. 95, 1072-1079. doi: $10.1002 /$ jsfa. 6800
Conflict of Interest Statement: The authors declare that the research was conducted in the absence of any commercial or financial relationships that could be construed as a potential conflict of interest.

Copyright (c) 2016 Falentin, Rault, Nicolas, Bouchard, Lassalas, Lamberton, Aubry, Marnet, Le Loir and Even. This is an open-access article distributed under the terms of the Creative Commons Attribution License (CC BY). The use, distribution or reproduction in other forums is permitted, provided the original author(s) or licensor are credited and that the original publication in this journal is cited, in accordance with accepted academic practice. No use, distribution or reproduction is permitted which does not comply with these terms. 